Title: Coupled FE-CFD Thermal Analysis for a Cooled Turbine Disk

Journal of Mechanical Engineering Science (Part C, Proc. IMechE)

\title{
Coupled FE-CFD Thermal Analysis for a Cooled Turbine Disk
}

$\begin{array}{cccc}\begin{array}{c}\text { *Umesh Javiya } \\ \text { umesh.javiya@rolls- }\end{array} & \begin{array}{c}{ }^{*} \text { John Chew } \\ \text { royce.com }\end{array} & \begin{array}{l}\text { i.chew@surrey.ac.uk } \\ \text { n.hills@surrey.ac.uk }\end{array} & \frac{\begin{array}{c}{ }^{* *} \text { Nick Hills } \\ \text { timothy.scanlon@roll }\end{array}}{\text { s-royce.com }}\end{array}$

* Rolls-Royce plc, PO Box 31, Derby DE24 8BJ, UK

**Thermo-Fluid Systems UTC, Faculty of Engineering and Physical Science, University of Surrey, Guildford, Surrey, GU2 7XH, UK

\section{Abstract}

This paper presents transient aero-thermal analysis for a gas turbine disk and the surrounding air flows through a transient slam acceleration/deceleration "square cycle" engine test, and compares predictions with engine measurements. The transient solid-fluid interaction calculations were performed with an innovative coupled finite element (FE) and computational fluid dynamics (CFD) approach. The computer model includes an aero-engine high pressure turbine (HPT) disk, adjacent structure, and the surrounding internal air system cavities. The model was validated through comparison with the engine temperature measurements and is also compared with industry standard standalone FE modelling. Numerical calculations using a 2D FE model with axisymmetric and 3D CFD solutions are presented and compared. Strong coupling between CFD solutions for different air system cavities and the FE solid model led to some numerical difficulties. These were addressed through improvement to the coupling algorithm. Overall performance of the coupled approach is very encouraging giving temperature predictions as good as a traditional model that had been calibrated against engine measurements.

Key Words: Gas turbine, Secondary air system, Turbine disk, Structure-Fluid interaction, Heat transfer, CFD, FEA, FE-CFD coupling 
Title: Coupled FE-CFD Thermal Analysis for a Cooled Turbine Disk

Journal of Mechanical Engineering Science (Part C, Proc. IMechE)

\section{INTRODUCTION}

The secondary air system of a gas turbine engine is designed to provide sufficient cooling for the components, manage bearing loads, seal bearing chambers, and prevent ingestion of hot mainstream gas into the turbine disk cavities. Metal temperatures must be limited to achieve required component lifetimes and temperature variation through the operating cycle affects turbine and compressor disks growth and achievable running clearances. However, bleeding cooling air from compressor stages to supply the internal air system incurs penalties in thermal efficiency. Hence, efficient use of the cooling air and robust design of the secondary air system requires a full understanding of the flow and heat transfer processes. In conventional industrial practice finite element (FE) analysis is used for heat transfer analysis to predict metal temperatures and thermo-mechanical stresses. In this method, the convective heat transfer is modelled using correlations based on benchmark experiments, previous engine modelling, standalone computation fluid dynamics (CFD) studies and/or engine test data. This requires significant human effort, possibly with the aid of optimisation tools, to match engine test data [1]. It is therefore attractive to develop automatic boundary condition treatment by fully integrating CFD in the thermal analysis.

With advances in parallel computation and numerical modelling there is a trend in turbomachinery design systems towards the "virtual" or "whole" engine simulation $[2,3]$. Recognizing the importance of aero-thermo-mechanical effects, coupling of FE component models with detailed CFD models is receiving considerable attention from the turbomachinery research community. Several researchers have demonstrated FECFD coupled analysis capability for heat transfer calculations in gas turbines [4 to 12]. Detailed validation of the coupled analysis approach was demonstrated by Javiya 
Title: Coupled FE-CFD Thermal Analysis for a Cooled Turbine Disk Journal of Mechanical Engineering Science (Part C, Proc. IMechE)

et al. [10] for a research test rig incorporating a model turbine disk with a pre-swirled cooling air delivery system. All these analyses used a single CFD domain with traditional convective boundary conditions applied on remaining boundaries of the FE model.

The main objectives of the present study were to provide a step forward in industrial scale automatic transient FE-CFD coupled analysis, and establish the validity of the approach through comparison with engine test data. The test case selected was the high pressure turbine (HPT) disk of a modern aero engine with all the surrounding air system flows modelled with CFD. Transient calculations were performed for a typical slam acceleration/deceleration "square cycle" engine test.

The algorithm for coupling the FE solution with the multiple fluid solutions is presented in section 2. A short description of the HPT FE model and the transient engine running cycle is given in section 3. Descriptions of the five CFD models that represent the flow surrounding the HPT disk are given in section 4. This section also describes the flow structures in the air system cavities. Results from the coupled calculations are presented and compared to the engine test data in section 5, and the main conclusions of the study are given in section 6 .

\section{COUPLED FE-CFD METHODOLOGY}

The convective time scale for the fluid domain will be order of $\mathrm{L} / \mathrm{U}$ (where, $\mathrm{U}$ and $\mathrm{L}$ are characteristic velocity and length scales) and the time scale for solid domain conduction will be of order $\mathrm{L}^{2} / \alpha$ (where, $\alpha$ is thermal diffusivity). Hence the time scale ratio of solid to fluid response is in the order of LU/ $\alpha$. With representative values of $U=200 \mathrm{~m} / \mathrm{s}, \alpha=10^{-5} \mathrm{~m}^{2} / \mathrm{s}$ (e.g. carbon steel), and $\mathrm{L}=0.1 \mathrm{~m}$, the time scale ratio is in the order of $2 \times 10^{6}$. For the calculation of metal temperatures and thermo- 
Title: Coupled FE-CFD Thermal Analysis for a Cooled Turbine Disk

Journal of Mechanical Engineering Science (Part C, Proc. IMechE)

mechanical stresses, it is reasonable to assume instant response of the flow due to changes in boundary conditions and resolve time accurate transient thermal behaviour of the solid domain only. Hence in this paper the transient coupled FE-CFD calculations are performed with steady state CFD solution in fluid domains and transient FE solution in the solid domains.

The transient coupled FE-CFD algorithm used is similar to that used by Verdicchio et al. [4], Illingworth et al. [5], Autef [6] and Sun et al. [7]. As illustrated in Figure 1, the coupled FE and CFD domain wall boundaries exchange heat flux and temperature iteratively at each time step until a user specified temperature convergence is reached. The FE-CFD coupling algorithm is programmed within the FE code and invokes an external CFD solver as necessary.

The coupled walls were provided with Dirichlet (temperature) boundary conditions on the fluid side and Neumann (heat flux) boundary conditions on the solid side. Instead of providing a pure heat flux, $q_{w}$ boundary condition on the solid FE domains, a heat transfer coefficient $h$, and fluid temperature $T_{f}$ were used such that $q_{w}=h\left(T_{f}-T_{w}\right)$. This results in a better convergence rate for the coupled iterative calculations (Verdicchio [4], Verstraete et al. [13]). Values of $h$ at each boundary node were calculated from two different CFD solutions for heat flux $q_{w}$ and $q_{w, 2}$, with different wall temperatures $T_{w}$ and $T_{w}+\Delta T_{w}$ using the following equation.

$$
h=\frac{q_{w}-q_{w, 2}}{\left(T_{w}+\Delta T_{w}\right)-T_{w}}=\frac{q_{w}-q_{w, 2}}{\Delta T_{w}}
$$

$\Delta T_{w}$ was chosen as $20 \mathrm{~K}$. The value of this small change in wall temperature only weakly affects the heat transfer coefficient, $h$.

To ensure the correct heat flux to the solid the fluid temperature is calculated as follows.

$$
T_{f}=\frac{q_{w}}{h}+T_{w}
$$


Title: Coupled FE-CFD Thermal Analysis for a Cooled Turbine Disk

Journal of Mechanical Engineering Science (Part C, Proc. IMechE)

It should be noted that the $\mathrm{h}$ is just a representative value for accelerating convergence of the coupled calculation. To reduce the computation time in transient calculations, $\mathrm{h}$ is estimated only once at the beginning of each time step. However, the iterative variation in the heat flux is adjusted by changing fluid temperature $T_{f}$ in equation (2) at each of the boundary nodes.

In the FE solid domain model, not all boundary conditions are given by the CFD solutions. Boundary conditions for surfaces not included in the CFD domains are specified using standard one dimensional convective boundary treatments such as streams and ducts as described by Dixon et al. [1]. These one-dimensional flow elements can also link with CFD inlet and outlet conditions allowing, for example, continuity of air total temperature between elements of the air system at each time point. The outlet CFD boundaries may also supply the swirl velocity to other flow elements, as illustrated in Figure 1.

Owing to the strong coupling of inlet and outlet fluid domain boundaries and the large number of coupled walls in the present model, numerical instabilities arose in the transient calculation. These were overcome using the following measures.

- A lower bound of $\mathrm{h}=200 \mathrm{~W} / \mathrm{m}^{2} \mathrm{~K}$ was applied. This avoids large changes in wall temperature between successive coupled iterations that can occur for small values of $h$.

- Under relaxation of heat flux in the coupling iterations was applied using the following equation,

$$
\text { - } \quad q_{w}^{k+1}=q_{w}^{k}+\beta \times\left(q_{w}^{k+1}-q_{w}^{k}\right)
$$

where, $\beta$ is a heat flux under relaxation parameter and $\mathrm{k}$ is the coupled iteration counter. 
Title: Coupled FE-CFD Thermal Analysis for a Cooled Turbine Disk

Journal of Mechanical Engineering Science (Part C, Proc. IMechE)

- Averaging of heat flux over the last few iterations in the CFD calculation was applied. This damped fluctuations of the heat flux observed at some locations, helping to avoid oscillations in the coupled solution.

Transient coupled analyses were carried out with the Rolls-Royce in-house FE code SC03 and two CFD solvers, Fluent and Hydra, were used as will be described below. The coupling algorithm was programmed as a plug-in to the FE code. More details of the coupling algorithm are given by Illingworth et al. [5], Autef [6], Sun et al. [7] and Verdicchio et al. [11]. In all CFD calculations, at least 550 steady state CFD iterations were performed for each fluid domain per coupled iteration. Averaging of the heat flux solution over the last 100 iterations was implemented within the Hydra solver. For the present study, implementation was modified to allow multiple CFD calculations to be performed in parallel.

\section{FE MODEL}

The test case selected was a Rolls-Royce three spool gas turbine engine. The model includes three rotating shafts corresponding to high, intermediate and low pressure (HP, IP and LP) turbine speeds. The FE model is shown in Figure 2. The model includes the HP turbine disk and blade, two HP compressor stages, the IP turbine shaft, the LP turbine shaft and surrounding stationary components. The components are coloured with the corresponding rotating speeds. All components were modelled as axisymmetric with appropriate modelling assumptions to account for three dimensional features. This followed industrial practice with features such as bolts, holes etc. modelled using an "equivalent wall thickness" where this was considered necessary

The solid domain discretisation was carried out within the SC03 code using 6 noded triangular elements. Transient non-linear thermal conduction equations in the 
Title: Coupled FE-CFD Thermal Analysis for a Cooled Turbine Disk Journal of Mechanical Engineering Science (Part C, Proc. IMechE)

FE code were solved with second order accuracy. At each time step, conduction equation residuals were checked for convergence. In addition, the time stepping error at each node of the solid domain was estimated as the maximum difference between a quadratic curve fitted through temperatures at three successive time steps and a linear curve fit between these time steps. If convergence criteria were not met then the current time-step results were discarded and the solution restarted with a smaller time step. Further description of the FE code thermal calculation process is given in references $[11,12]$.

In typical engine tests, the thermocouple uncertainties are about $5 \mathrm{~K}$ for steady and $10 \mathrm{~K}$ for transient conditions [1]. Hence, the time stepping accuracy was specified as $2.5 \mathrm{~K}$ in the coupled solutions. This was reduced to $1.25 \mathrm{~K}$, for $2 \mathrm{D}$ calculations with Hydra when oscillations were observed at some points of the solution in this case.

In the original "stand alone" FE thermal model the convective heat transfer on the cavity walls was modelled with one-dimensional duct, stream and void boundary conditions as described by Dixon et al. [1]. This model was calibrated against engine temperature measurements and is referred to as the "baseline" FE model below. In addition to convective heat transfer, the model also accounts for radiative heat exchange where appropriate.

Thermal calculations were performed for a slam acceleration/deceleration "square cycle" engine test defined in terms of the HP turbine shaft speed in Figure 3. The cycle was described by interpolation of specified conditions at 19 "ramp points". The cycle started with a ramp from stand-still to a low engine power condition (ramp point 1 to 2 ), followed by short acceleration and deceleration steps (ramp points 3 to 6). It was allowed to stabilize from ramp point 6 to 7 before accelerating to maximum power (ramp points 7 to 12 ). Following stabilisation at maximum power the engine 
Title: Coupled FE-CFD Thermal Analysis for a Cooled Turbine Disk Journal of Mechanical Engineering Science (Part C, Proc. IMechE)

decelerated (ramp points 13 to 18) and stabilized at low power condition (ramp point 18 to 19$)$. The entire cycle took around $8000 \mathrm{~s}$ or 2.2 hours. The sections of the cycle at constant rotation speed are described as "steady ramps" and the acceleration or deceleration ramps are referred to as "transient ramps". For transient calculations, all these ramp points were specified with the necessary rotation speeds, mass flow rates, pressures and temperatures based on the engine pressure measurements, using best understating of one-dimensional air system calculations.

In the coupled FE-CFD analysis flow in the five secondary air cavities shown in Figure-2 were modelled using CFD which supplied convective boundary conditions for the FE solution. The cavities are connected through labyrinth seals and these seal clearances vary as components deflect during the engine test cycle. This was accounted for through variation of the mass flow rates through the seals through the cycle. This allowed use of established correlations for seal flow rates.

\section{CFD MODELS}

As mentioned earlier, the coupling algorithm uses steady state CFD calculations. Changes in rotation speed and mass flow rate are accounted for by creating three CFD models for each of the five cavities. These three conditions correspond to low, maximum and intermediate power and were matched to operating conditions at ramp points 3, 4 and 9 in Figure 3. Referring to the Figure 3, CFD-1 models were used during ramps 1 to 3,6 to 7 and 18 to 19 , CFD-2 models were used during ramps 8 to 10 and 15 to 17 and CFD-3 models were used during ramps 4 to 5 and 11 to 14 . The transient ramps ( 3 to 4,5 to 6,7 to 8,10 to 11,14 to 15 and 17 to 18 ) used interpolation between appropriate CFD models. Inlet and outlet mass flow rates were fixed in all fluid domains but the inlet and outlet temperatures varied, as described earlier, by coupling with the one-dimensional flow elements. 
Title: Coupled FE-CFD Thermal Analysis for a Cooled Turbine Disk

Journal of Mechanical Engineering Science (Part C, Proc. IMechE)

Two CFD codes were used; the commercial code FLUENT [15] and RollsRoyce's in house Hydra CFD code [14]. In FLUENT, the Reynolds-averaged NavierStokes (RANS) and turbulence model equations were solved in a segregated manner using the SIMPLE (Semi-Implicit Method for Pressure-Linked Equations) algorithm. In Hydra, the equations were solved in a coupled manner using density based algorithms. In all cases, compressible flow was assumed using the ideal gas and Sutherland's laws for the density and viscosity of air and including frictional effects in the energy equation. Turbulence was modelled with the standard k- $\varepsilon$ model with wall functions. Previous evaluations of the two CFD codes have shown that they give very similar results with resolved near-wall treatments, but differences can arise when using the k- $\varepsilon$ turbulence model with wall functions due to different implementations of the wall functions [16].

Second-order discretisation was used in all cases except the 3D pre-swirl cavity model which will be explained below. Hydra, axisymmetric (2D) solutions were obtained with a thin $0.4^{\circ}$ periodic sector, whereas with FLUENT axisymmetric solutions were obtained more directly using a single mesh plane.

All axisymmetric and 3D fluid domains were discretised with quadratic and hexahedral cells respectively generated using the ICEM-CFD code [17]. The mesh sizes were approximately 3,000 to 700,000 cells depending on the domain size. Exhaustive mesh dependency studies were not carried for the full transient coupled calculations. However, mesh density in all domains was consistent with previous experience and CFD evaluations against experimental data, as shown for example, by Javiya et al. $[10,16]$. Near wall mesh y+ values for maximum power conditions are shown in Figure-4 for 3D solutions. These were in the accepted range (30 to 150) over most of the coupled walls in all three initial CFD models of all five cavities. 
Title: Coupled FE-CFD Thermal Analysis for a Cooled Turbine Disk

Journal of Mechanical Engineering Science (Part C, Proc. IMechE)

Considering the complexity of the geometries and time spent in creating these meshes, the $y+$ values were considered acceptable. There were significantly higher $y+$ values in the bucket grooves of the pre-swirl outer cavity model in Figure-4. However, the bucket groove walls were not a part of coupled calculations. Similar y+ values were achieved for the low and intermediate power CFD models.

Boundary conditions were specified corresponding to the non-dimensional parameters as given in Table-1. The rotational Reynolds number, $R e_{\phi}\left(=\rho r_{p}^{2} \Omega_{H P} /\right.$ $\mu$ ) was calculated based on pre-swirl nozzle radius $\left(r_{p}\right)$, HPT rotor speed $\left(\Omega_{H P}\right)$, density $(\rho)$ and viscosity $(\mu)$ at pre-swirl inlet conditions. The non-dimensional flow rate, $\left(\lambda_{T}\right)$ for each inlet was calculated from the definition $\lambda_{T}=C_{w} \times R e_{\phi}^{-0.8}$ and $C_{w}=\dot{m} / \mu r_{p}$. The mass flow parameter $C_{w}$ was calculated using the inlet flow rate $\dot{m}$, pre-swirl nozzle radius $r_{p}$ and properties at inlet conditions. The inlet names given in the table correspond to those used in figures below. Mass flow rate and either flow angle or swirl velocity were specified at all inlets. Pressure was specified at one outlet in each domain and mass flow rate was specified at other outlets. Representative inlet temperatures were used in the initial CFD calculations. Inlet temperatures varied during the coupled calculations but the mass flow rates and pressures at all boundaries were constant. At all inlets boundary conditions for $k$ and $\varepsilon$ assumed 5\% turbulence intensity and a $1 \mathrm{~mm}$ turbulence length scale. Details of the CFD solutions are given in the following subsections.

\subsection{Pre-swirl chamber}

Axisymmetric and 3D models for the pre-swirl chamber are shown in Figure-5. The 3D model sector's periodicity corresponds to the number of high pressure turbine blades. The chamber has two inlets, the main pre-swirl nozzle inlet and inner labyrinth seal flow. In both axisymmetric and 3D models, the inlets were represented by an 
Title: Coupled FE-CFD Thermal Analysis for a Cooled Turbine Disk

Journal of Mechanical Engineering Science (Part C, Proc. IMechE)

axisymmetric slot. In the 3D model, the cover plate and bucket groove cavity details were included, with three outlets (Outlet-1 to 3) for the blade cooling passages as shown in Figure-5b. In the axisymmetric model bucket groove details were not included and modelled with single outlet. Both models included the leakage flow through cover plate (Outlet-4) and outer labyrinth seal leakage flow (Outlet-5). The receiver holes were represented as a slot in the axisymmetric model but modelled as a 3D feature in 3D model. Rotating walls are red and stationary walls are blue this figure. There were three outlets in the axisymmetric model and five outlets in the 3D model. The mass flow inlet conditions were specified with fixed flow angle corresponding to the nozzle angle at the main pre-swirl inlet and with fixed swirl velocity at the inner labyrinth seal inlet. Outlet-3 was selected for specification of the pressure.

For 3D calculations in Hydra at the maximum power condition, some convergence issues were encountered when using the second order discretisation scheme in the transient coupled calculations. The flow inside the cavity fluctuated significantly. It may be noted that a similar 3D pre-swirl cavity case including the nozzle but without the complex bucket groove geometry was successfully simulated with Hydra by Javiya et al. [18]. It was considered that the unsteady effects here were associated with inclusion of the receiver holes and bucket grooves. Hence, it was decided to use first order calculations, which converged well, in this cavity only. The second order scheme was retained for the axisymmetric model.

Figures 5 and 6 show computed flow structures from the axisymmetric and 3D calculations for the maximum power condition. Similar flow structures were obtained at other operating conditions. It can be observed that there are two vortices in the main pre-swirl chamber and one vortex in the cover plate cavity. 3D features in the 
Title: Coupled FE-CFD Thermal Analysis for a Cooled Turbine Disk

Journal of Mechanical Engineering Science (Part C, Proc. IMechE)

3D model create a more complex flow structure in the cover plate cavity. Significant flow separation was observed in the receiver holes and bucket grooves in Figure 6.

Radial profiles of the circumferentially averaged swirl velocity on two axial planes are compared for axisymmetric and 3D models in Figure-7. Either side of the nozzle radius $r / r_{p} \sim 0.84 \mathrm{~m}$, the non-dimensional swirl velocity is almost constant, which shows forced vortex type behaviour. It can be seen that the axisymmetric calculations from Fluent and Hydra agree well in both the main pre-swirl and cover plate cavities. In the main pre-swirl cavity, the swirl velocity peak is broader in the $3 \mathrm{D}$ case with Hydra than the axisymmetric case. This is may be because the first order calculation in the 3D case is more diffusive than the second order scheme and hence the nozzle jet becomes more spread inside the cavity. The swirl velocities inside the cover plate cavity for the 3D case are significantly different from the axisymmetric calculations. This can be attributed to the swirl velocity pick-up of the air through the rotating holes in the 3D model.

\section{$\underline{4.2}$ Pre-swirl inner cavity}

The axisymmetric pre-swirl inner cavity model is shown in Figure-8. There are no $3 \mathrm{D}$ features in pre-swirl inner cavity hence the 3D domain was essentially an axisymmetric model covering a 0.4 degree periodic sector. For this domain there were two inlets; one for sealing flow from the inner labyrinth seal and the second for the side leakage. The only outlet was to the outer labyrinth seal. Boundary conditions were specified mass flow rate with constant whirl velocity at the inner seal, mass flow rate and flow normal to boundary at the second inlet, and pressure at the outer seal.

The axisymmetric Fluent solution for the maximum power condition is shown by the computed streamlines in Figure-8a. The flow inside this cavity is similar to classical stator-rotor cavity flow as reported, for example, by Dorfman [19]. There is a 
Title: Coupled FE-CFD Thermal Analysis for a Cooled Turbine Disk

Journal of Mechanical Engineering Science (Part C, Proc. IMechE)

central rotating core and air is pumped radially outward at the HPT disk wall due to centrifugal action and radially inward at the static wall. The core rotates at almost $26 \%$ of the HPT disk speed. This is less than the enclosed stator-rotor cavity flow value of approximately 0.45 [19] because of the superimposed radial inflow through the inner labyrinth seal and increased area of the stator wall. The flow structures for the other two operating conditions were similar to that at the maximum power condition. The swirl velocities in Figure-8b show reasonable agreement between Hydra and Fluent solutions.

\subsection{Turbine rear cavity}

The turbine rear cavity axisymmetric and 3D models are shown in Figure 9. In the 3D model, bolts were included near the inner radius. As in the main pre-swirl chamber, the nozzles on the rear drive arm were modelled as an axisymmetric slot (Rinlet-1) to enable steady state calculations. Periodicity of the 3D domain corresponds to the number of bolts. Both axisymmetric and 3D models contained only one inlet representing the pre-swirl nozzles, and two outlets, representing the inner labyrinth seal (Routler-2) and to the main gas path through the HP disk rear rim seal (Routlet-1). In practice, there may be some hot gas ingestion from the main annulus into the outer damping cavity. However, this effect was not modelled here. To avoid any reverse flow at the outlet in the CFD calculations the Routlet-1 boundary was closed to $55 \%$ of its nominal static value. Constant mass flow rate at a flow angle corresponding to the nozzle angle was specified at the inlet, mass flow was specified at the inner radius seal, and pressure was specified at the outer radius opening to the main gas path.

Calculated swirl velocities are plotted on four different lines in Figure-10 for the maximum power condition. Again, the axisymmetric Fluent and Hydra calculations 
Title: Coupled FE-CFD Thermal Analysis for a Cooled Turbine Disk

Journal of Mechanical Engineering Science (Part C, Proc. IMechE)

matched well. The swirl ratio $\left(v_{\phi, r} / \Omega r\right)$ decreases with increasing radius up to $r / r_{p}<$ 0.8 , suggesting free vortex type behaviour. There are significant differences in swirl velocity between the axisymmetric and 3D models, especially for $r / r_{p}<0.8$. This is due to significantly different flow structures predicted by the two models, as shown in Figure 11. In the axisymmetric solution two main vortices were formed, rotating in opposite directions, as shown in Figure11a. In the 3D solution two main vortices were formed, rotating in the same direction, as shown in Figure-11b. It was also found that the steady Hydra axisymmetric model can predict both flow structures when different initial velocity and pressure distributions were specified. However, it was found in the coupled FE-CFD study that these significant flow differences did not greatly affect the HPT disk temperature predictions. This may be due to weak dependency of the boundary layer on the HPT rear face on the core region flow vortex structures.

\subsection{Drive cone cavity}

The drive cone cavity axisymmetric and 3D models are shown in Figure 12. The bolts on the stationary drive cone casing were only included in the $3 \mathrm{D}$ models. Periodicity of the 3D domain corresponded to the number of bolts. In this cavity, there was only one inlet, which is the bleed flow from the final stage of the high pressure compressor, and one outlet to the labyrinth seal. Mass flow and swirl velocity were specified at the inlet, and pressure was specified at the outlet.

Calculated swirl velocities for the maximum power condition are plotted on two different axial lines in Figure 13. Again Fluent and Hydra predicted similar results for the axisymmetric calculations. The solution for the interior of this cavity showed almost forced vortex behaviour which is consistent with previous experimental and numerical studies by Long et al. [20] and Tham et al. [21]. In the axisymmetric solutions, the air rotated at almost 0.5 and 0.39 times the speed of the HP shaft 
Title: Coupled FE-CFD Thermal Analysis for a Cooled Turbine Disk

Journal of Mechanical Engineering Science (Part C, Proc. IMechE)

upstream and downstream of the bolt location, respectively. In the 3D solution, the air rotated at almost 0.41 and 0.16 times the speed of the HP shaft at upstream and downstream of the bolt location, respectively. The differences in swirl velocities were mainly due to the bolt drag in the 3D model. The stationary bolts reduce the swirl in the core and hence increase rotor drag and frictional heat generation in the cavity. This effect was not captured in the axisymmetric calculations. This may significantly affect the heat transfer calculations and air temperature in this cavity and further downstream in the pre-swirl cavities.

\section{$\underline{4.5}$ Bore cavity}

The bore cavity axisymmetric and 3D models are shown in Figure 14. All three rotating shafts are present in this cavity. Walls are coloured red, green, brown and blue corresponding to HP, IP, LP shafts and static components, respectively. To allow steady calculations (in a rotating reference frame corresponding to HP shaft speed), only one $3 \mathrm{D}$ feature, holes in the rear drive arm, was resolved in the $3 \mathrm{D}$ model. The holes in the IP shaft were modelled as axisymmetric slots with the same opening areas as the holes and the effects of bolts were neglected in both axisymmetric and 3D models.

The flow direction through the outer radius labyrinth seal on the rear drive arm (Binlet-seal-1) reversed during the flight cycle. Hence, it is marked with both blue and red arrows in Figure-14. At the low power condition, the flow through this seal was outward and during the maximum power condition it was into the cavity. Hence, this boundary was specified as an outlet with specified flow rate at the low and intermediate power conditions, and an inlet boundary at the maximum power condition. Inlet flow rates and swirl velocities were specified at the remaining 5 inlets, and outlet pressure was specified at the main outlet. 
Title: Coupled FE-CFD Thermal Analysis for a Cooled Turbine Disk

Journal of Mechanical Engineering Science (Part C, Proc. IMechE)

Calculated swirl velocities on various lines inside the bore cavity are shown in Figure 15 for the maximum power condition. Some differences were observed between the Fluent and Hydra results for the axisymmetric case on line- 1 in the front bore cavity where the flow is expected to be close to a forced vortex. However, it should be noted that steady flow modelling might not be adequate for this "rotorrotor" type of cavity. The flow in this cavity may be subject to large scale unsteady oscillations as, for example, discussed by Owen and Pincombe [22], Bohn et al. [23] and Sun et al. [24]. The Hydra 3D model solution was similar to the axisymmetric case except on "Line-2" and for $x / x_{o}>1.04$, in Figure 15. This was due to the presence of the holes in the $3 \mathrm{D}$ model. The flow rotates at a swirl ratio $\left(v_{\phi, r} / \Omega_{\mathrm{NH}} r\right)$ almost equal to 1, exhibiting forced vortex type behaviour in both the front and rear cavities, and so convective heat transfer to the disk in these regions is expected to be weak.

\section{TEMPERATURE PREDICTION}

Results are presented for three different coupled FE/CFD models; 2D-2D coupling with Fluent, 2D-2D coupling with Hydra and 2D-3D coupling with Hydra. These results are compared with the baseline (uncoupled) FE model and engine test data. Both 2D-2D coupled calculations were performed for the full transient cycle. The 2D-3D coupled solution was stopped after the first acceleration and deceleration step (up to ramp point 7 in Figure 3).

The coupled calculations were performed on two PC clusters with 2.6 GHz AMD 128 nodes (with 4 cores on each node), and $3.2 \mathrm{GHz}$ Intel 48 nodes (with 8 cores on each node). Both clusters had fast Myrinet networking. The 2D-2D calculations took about 25 days using Hydra and about 7 days using Fluent. This reflects the tendency of some Hydra solutions to oscillate rather than fully converge to a steady solution. Overall time for the 2D-3D computation using 260 cores was about 3.5 months up to 
Title: Coupled FE-CFD Thermal Analysis for a Cooled Turbine Disk

Journal of Mechanical Engineering Science (Part C, Proc. IMechE)

ramp point 7 . This solution was significantly affected by numerical oscillations, increasing the computing time. Further work by Ganine et al. [9] has led to the improvements in the algorithm, but these were not available for this study.

Predicted temperatures were recorded at reference points corresponding to engine test thermocouple positions. A typical temperature result at one of the reference point (TCF0) is shown against test time in Figure 16. The figure also shows the engine test data. These measurements were obtained from an engine development test for the transient cycle in figure 3, as discussed, for example, by Dixon et al. [1]. In this plot, it is difficult to observe the difference between calculated and engine test temperatures. Hence, the calculated temperatures are presented as differences between calculated and measured temperatures in sub-section 5.1 below. Full field temperature distributions are presented in sub-section 5.2.

\subsection{Comparison with measurements}

Thermocouple locations and predicted temperatures minus measured temperatures divided by high pressure compressor (HPC) exit temperature (T30) at high power against test time are shown in Figures 17, 18 and 19. High frequency fluctuations in these temperature difference plots originate from the measured data.

Figure 17 shows results at the front of the turbine disk at five thermocouple locations TCF1 to TCF5. All three coupled model predictions showed accuracy of \pm 0.01 ( $\pm 1 \%$ of $\mathrm{T} 30)$ compared to measurement at stabilized conditions, except position TCF3 which may be due to its proximity to the cover plate holes. For the acceleration and deceleration thermal transient steps all three coupled models and the baseline models were not well predicting the measurements. In the bucket groove region (ref. points TCF1 and TCF2), all three coupled models predicted similar results to the baseline uncoupled model. The 2D-3D coupled model generally predicted 
Title: Coupled FE-CFD Thermal Analysis for a Cooled Turbine Disk

Journal of Mechanical Engineering Science (Part C, Proc. IMechE)

higher temperatures than 2D-2D coupled models owing to inclusion of the nonaxisymmetric features as discussed earlier. At location TCF3, Hydra calculations showed higher temperature predictions than those with Fluent. This is attributed to the different wall function implementations in the two codes combined with pre-swirl jet impingement on the rotating surface, as was indicated in an the earlier study by Javiya et al. [16].

Considering the uncertainties due to boundary conditions and other factors the coupled solutions are considered to compare well to the baseline model which has been adjusted to give some alignment with measurements.

Figure 18 shows results for four thermocouple locations TCR1 to TCR4 on the turbine disk rear face. Calculations are within $\sim \pm 0.01$ of T30 compared to the engine test data for the steady conditions. Greater differences between the models occur at inner radii where the effects of non-axisymmetric features and flow structure differences are likely to be higher. However these differences are not sufficient to identify which of the steady CFD flow structures in the rear cavity is more realistic. At locations TCR3 and TCR4 the transient ramp responses following the decelerations are closer to the engine test data than the baseline model. Conversely the baseline model gave a slightly better match with measurement for the transient following the accelerations.

Figure 19 shows results for five thermocouples TCB1 to TCB5 inside the drive cone and bore cavities. At locations TCB2, TCB4 and TCB5, the 2D-3D calculations for the low power condition are generally closer to the measurements than the baseline and 2D-2D calculations.

Oscillations in the 2D-2D Hydra solution at TCB1 may be associated with convergence difficulties in the CFD solutions. The relatively wide spread in results at 
Title: Coupled FE-CFD Thermal Analysis for a Cooled Turbine Disk

Journal of Mechanical Engineering Science (Part C, Proc. IMechE)

location TCB3 may be associated with the flow complexity in the rear bore cavity.

Note, however, that the performance of the predictive coupled FE-CFD models is comparable to the baseline model.

Considering the overall results, the stabilized coupled model predictions were as good as the baseline model except at a few locations. For transient conditions all the models show greater deviation from the test data and at some locations, the CFD based models performed worse than the baseline model.

\subsection{Temperature contour plots}

Instantaneous temperature contour plots at $\mathrm{t}=2700 \mathrm{~s}$, corresponding to stabilized low power conditions, from the baseline model and the 2D-2D and $2 \mathrm{D}-3 \mathrm{D}$ coupled solutions using Hydra are shown in Figure 20. Stabilised maximum power temperature contours for the baseline and 2D-2D coupled solutions are shown in figure 21. The contour plot range is normalised using the temperature rise across the HP compressor. Similar conclusions can be drawn from the contour plots as from the reference point results. The coupled calculation temperatures differ most noticeably from the baseline model results near the drive cone cavity and rear cavity drive arm as marked with boxes in the figures. Some significant differences occur in regions such as the rear cavity drive arm where there are no thermocouples. In these regions the coupled model may be preferred as traditional thermal models rely on matching to thermocouple data. The neglect of non-axisymmetric geometric features clearly has some effect as shown by comparison of results from the two coupled models.

\section{CONCLUSIONS}

Coupled FE-CFD turbine disk temperature predictions have been presented and compared with engine measurements for a full transient engine test cycle. This demonstrates the potential for practical application of this method, and is a step 
Title: Coupled FE-CFD Thermal Analysis for a Cooled Turbine Disk

Journal of Mechanical Engineering Science (Part C, Proc. IMechE)

towards virtual engine modelling as discussed by Chew and Hills [2]. Accuracy of the CFD based model was comparable to that of an industrial standard baseline FE model that had been adjusted to match engine data. Significant differences between the baseline and coupled models were found in regions where there are no thermocouple data. In such regions the coupled model may be preferred.

The test case considered highlighted some areas where the original coupling algorithm needed improvement. Measures were taken to control numerical instabilities associated with strong coupling between multiple fluid domains and heat transfer in the solid components. Significant improvements were achieved by limiting the heat transfer coefficient used, under relaxation of heat flux exchange between solid and fluid domains, and averaging the last few iterations for heat flux in the CFD solutions. Further work on improving the coupling algorithm has been reported by Ganine et al. [9].

The flow predictions from axisymmetric and 3D CFD models were discussed in some detail for the five cavities considered. As was expected from previous studies, some complex flow phenomena occur and the CFD models were limited in some respects. For example, 2D CFD solutions for the turbine rear cavity exhibited two distinct flow structures depending on the initialization of the flow solution. However comparison of predicted temperatures with engine test data could not distinguish which, if either, of the CFD calculations better represented engine behaviour.

Comparison of results from axisymmetric and 3D CFD models confirmed that 3D geometrical features such as bolt heads and holes could significantly affect the predictions.

To economise on computing requirements all CFD solutions assumed steady flow and the standard k- $\varepsilon$ turbulence model with wall functions. Unsteadiness was thought 
Title: Coupled FE-CFD Thermal Analysis for a Cooled Turbine Disk

Journal of Mechanical Engineering Science (Part C, Proc. IMechE)

to be significant in some cases, and this was associated with convergence difficulties

for some steady CFD solutions. The standard k- $\varepsilon$ model has some limitations for applicability in all cavities as pointed out in previous studies. Better results might be obtained in some regions with resolved near-wall treatments but this would significantly increase computation requirements.

It is expected that current and future work will improve the FE-CFD coupling algorithm, allowing further exploitation of the method in industrial design and analysis. Increasing computer power will allow use of more accurate CFD models, but a variety of models will be needed in balancing accuracy and completeness of models against computational cost. As shown by Amirante et al. [8] the FE-CFD coupling can be extended to include component deflections, allowing improved estimates of seal clearances and air system flow rates through the engine cycle. Such aero-thermomechanical analysis is expected to lead to further understanding and improved transient thermal modelling. Sensitivity of temperature predictions to uncertainties and inaccuracies in boundary conditions will also be of considerable interest, 
Title: Coupled FE-CFD Thermal Analysis for a Cooled Turbine Disk

Journal of Mechanical Engineering Science (Part C, Proc. IMechE)

\section{ACKNOWLEDGEMENTS}

The authors are grateful to the DHPA-EPSRC scholarship program and RollsRoyce for financial support of this work, and to colleagues for helpful comments. The authors gratefully acknowledge the permission of Rolls-Royce plc to publish this paper. 
Title: Coupled FE-CFD Thermal Analysis for a Cooled Turbine Disk

Journal of Mechanical Engineering Science (Part C, Proc. IMechE)

\section{Nomenclature}

\section{NOMENCLATURE}

$C_{w} \quad$ Non-dimensional mass flow rate (-)

$k \quad$ thermal conductivity $((\mathrm{W} /(\mathrm{mK}))$

$L \quad$ characteristic length scale (m)

$\dot{m} \quad$ mass flow rate $(\mathrm{kg} / \mathrm{s})$

$q \quad$ heat flux $\left(\mathrm{W} / \mathrm{m}^{2}\right)$

$U \quad$ characteristic velocity scale $\left(\mathrm{m}^{2}\right)$

$\mathrm{T}$ temperature $(\mathrm{K})$

$h \quad$ heat transfer coefficient $\left(\mathrm{W} /\left(\mathrm{m}^{2} \mathrm{~K}\right)\right)$

$r \quad$ radius $(\mathrm{m})$

$r_{p} \quad$ pre-swirl nozzle radius (m)

$R e_{\phi} \quad$ Rotational Reynolds number (-)

$\mathrm{t} \quad$ time $(\mathrm{sec})$

$v_{\phi, r} \quad$ swirl velocity at radius $\mathrm{r}(\mathrm{m} / \mathrm{s})$

$x \quad$ axial position (m)

$x_{p} \quad$ pre-swirl nozzle outlet axial position (m)

$\mathrm{y}+\quad$ non-dimensional wall distance (-)

\section{Greek}

$\alpha \quad$ thermal diffusivity $\left(\mathrm{m}^{2} / \mathrm{s}\right)$

$\beta \quad$ relaxation coefficient

$\rho \quad$ density $\left(\mathrm{kg} / \mathrm{m}^{3}\right)$

$\mu \quad \operatorname{viscosity}(\mathrm{kg} /(\mathrm{m} \mathrm{s}))$

$\lambda_{T} \quad$ through flow parameter (-)

$\Omega \quad$ rotation speed ( $\mathrm{rad} / \mathrm{s})$ 
Title: Coupled FE-CFD Thermal Analysis for a Cooled Turbine Disk

Journal of Mechanical Engineering Science (Part C, Proc. IMechE)

$\Omega_{\mathrm{NL}} \quad$ Low pressure turbine rotation speed ( $\left.\mathrm{rad} / \mathrm{s}\right)$

$\Omega_{\mathrm{NI}} \quad$ Intermediate pressure turbine rotation speed ( $\left.\mathrm{rad} / \mathrm{s}\right)$

$\Omega_{\mathrm{NH}} \quad$ High pressure turbine rotation speed ( $\left.\mathrm{rad} / \mathrm{s}\right)$

$\Delta \quad$ difference

\section{Superscripts}

$\mathrm{k} \quad \mathrm{k}^{\text {th }}$ number of coupled iteration

$\mathrm{n} \quad \mathrm{n}^{\text {th }}$ number of time step

\section{Subscripts}

2 for second domain

26 at high pressure compressor entry

30 at high pressure compressor exit

cal calculated value

f defined on fluid

inlet defined on inlet condition

max maximum condition

meas measured value

NL defined on Low pressure turbine

NI defined on Intermediate pressure turbine

$\mathrm{NH}$ defined on High pressure turbine

$p \quad$ at pre-swirl nozzle radius

ref reference condition

w defined on wall 
Title: Coupled FE-CFD Thermal Analysis for a Cooled Turbine Disk Journal of Mechanical Engineering Science (Part C, Proc. IMechE)

\section{REFERENCES}

1. Dixon, J.A., Verdicchio, J.A., Benito, D., Karl, A. and Tham, K.M., 2004. Recent developments in gas turbine component temperature prediction methods, using computational fluid dynamics and optimization tools, in conjunction with more conventional finite element analysis techniques. Proceedings of the Institution of Mechanical Engineers, Part A: Journal of Power and Energy, 218, pp.241-55.

2. Chew, J., and Hills, N., 2009. "Computational fluid dynamics and virtual aeroengine modelling". Proceedings of the Institution of Mechanical Engineers, Part C: Journal of Mechanical Engineering Science, 223(12), pp. 2821-2834.

3. Chew, J., and Hills, N., 2007. "Computational fluid dynamics for turbomachinery internal air systems". Philosophical Transactions of the Royal Society, 365, pp. $2587-2611$.

4. Verdicchio, J., Chew, J., and Hills, N., 2001. "Coupled fluid/solid heat transfer computation for turbine disks", ASME Paper No. 2001-GT-0205.

5. Illingworth, J., Hills, N., and Barnes, C., 2005. "3D fluid-solid heat transfer coupling of an aero-engine pre-swirl system". ASME Paper No. 2005-GT68939.Amirante, D., and Hills, N., 2010. “Thermo-mechanical FEA/CFD coupling of an interstage seal cavity using torsional spring analogy”. ASME Paper No. 2010-GT-2268

6. Autuf, V., 2007. Aerothermal Modelling for axial Turbines. Ph.D. Thesis, University of Surrey, UK.

7. Sun, Z., Chew, J., Hills, N., Volkov, K., and Barnes, C., 2010. "Efficient finite element analysis/computational fluid dynamics coupling for engineering applications". Journal of Turbomachinery, 132, pp. 031016-1-03101 6-9. 
Title: Coupled FE-CFD Thermal Analysis for a Cooled Turbine Disk Journal of Mechanical Engineering Science (Part C, Proc. IMechE)

8. Amirante D, Hills NJ, Barnes CJ. (2012) 'Thermo-Mechanical Finite Element Analysis/Computational Fluid Dynamics Coupling of an Interstage Seal Cavity Using Torsional Spring Analogy'. Journal of Turbomachinery, 134 (5).

9. Ganine V, Javiya U, Hills N, Chew J. (2012) 'Coupled fluid-structure transient thermal analysis of a gas turbine internal air system with multiple cavities'. Journal of Engineering for Gas Turbines and Power, 134 (10)

10. Javiya U, Chew J, Hills N, Dullenkopf K, Scanlon T., 2013, "Evaluation of computational fluid dynamics and coupled fluid-solid modeling for a direct transfer pre-swirl system", Journal of Engineering for Gas Turbines and Power, $135(5)$

11. Verdicchio, J.A., 2001. The Validation and Coupling of Computational Fluid Dynamics and Finite Element Codes for Solving Industrial Problems. Ph.D. Thesis, University of Sussex

12. Javiya, U., 2012. Aero-Thermal Analysis of Turbine Disc and Surrounding Flows. Ph.D. Thesis, University of Surrey, UK.

13. Verstraete, T., Alsalihi, Z. and Braembussche, V.d., 2006. Numerical study of the heat transfer in micro gas-turbines. ASME Paper No. GT2006-90161

14. Moinier, P., 1999. Algoritham developements of unstructured viscous flow solver. Ph.D. Thesis, University of Oxford, UK Moinier, 1999

15. Fluent Manual, 2006. Fluent 6.3 User Manual. Ansys Inc.

16. Javiya U, Chew JW, Hills NJ, Zhou L, Wilson M, Lock GD. (2011) 'CFD analysis of flow and heat transfer in a direct transfer pre-swirl system'. Journal of Turbomachinery, 134 (3) 
Title: Coupled FE-CFD Thermal Analysis for a Cooled Turbine Disk

Journal of Mechanical Engineering Science (Part C, Proc. IMechE)

17. ICEMCFD manual, ICEMCFD 13 Unser manual. Ansys Inc. 2011

18. Javiya, U., Chew, J.W., Hills, N. and Scanlon, T., 2011. A comparative study of cascade vanes and drilled nozzle designs for pre-swirl. ASME Paper No. GT2005-46006

19. Dorfman, L.A., 1963. Hydrodynamic Resistance and Heat Loss of Rotating Solids. Oliver and Boyd, UK

20. Long, C. A., Turner, A. B., Kais, G., Tham, K. M., \& Verdicchio, J. A. (2003). Measurement and CFD Prediction of the Flow Within an HP Compressor Drive Cone. Journal of Turbomachinery , 125, 165-172

21. Tham, K.M., Long, C.A., Turner, A.B. and Dixon, J.A., 2003. Computation of the flow in a H.P. compressor drive cone cavity. IGTC Conference, Tokyo, Paper No. TS-024

22. Owen, J. M., and Pincombe, J. R., 1979 "Vortex breakdown in a rotating cylindrical cavity” Journal of Fluid Mechanics, 90 (1), pp. 109-127

23. Bohn, D., Ren, J. and Tuemmers, C., 2006. Investigation of the unstable flow structure in a rotating cavity. ASME Paper no. GT2006-90494

24. Sun, Z., Lindblad, K., Chew, J.W. and Young, C., 2007. LES and RANS Investigations Into Buoyancy-Affected Convection in a Rotating Cavity with a Central Axial Through flow. Journal of Engineering for Gas Turbines and Power, 129(2), pp.318-25. 
Title: Coupled FE-CFD Thermal Analysis for a Cooled Turbine Disk Journal of Mechanical Engineering Science (Part C, Proc. IMechE)

$\underline{\text { Tables }}$

\begin{tabular}{|c|c|c|c|c|c|}
\hline & & & $\begin{array}{l}\text { Low } \\
\text { Power }\end{array}$ & $\begin{array}{c}\text { Intermediate } \\
\text { Power } \\
\end{array}$ & $\begin{array}{c}\text { Maximum } \\
\text { Power }\end{array}$ \\
\hline & \multicolumn{2}{|l|}{ Ramp points } & 3 & 10 & 4 \\
\hline & \multicolumn{2}{|l|}{$\mathrm{Re}_{\phi}$} & $1.436 \mathrm{E}+07$ & $4.033 \mathrm{E}+07$ & $2.731 \mathrm{E}+07$ \\
\hline Cavity name & Boundary name & & & & \\
\hline \multirow{2}{*}{$\begin{array}{c}\text { Pre-swirl Inner } \\
\text { cavity }\end{array}$} & Pinner-inlet-1 & $\lambda_{T}$ & 0.165 & 0.120 & 0.123 \\
\hline & Pinner-inlet-2 & $\lambda_{T}$ & 0.002 & 0.002 & 0.002 \\
\hline \multirow{2}{*}{$\begin{array}{c}\text { Pre-swirl Outer } \\
\text { cavity }\end{array}$} & Pouter-inlet-1 & $\lambda_{T}$ & 0.375 & 0.573 & 0.638 \\
\hline & Pouter-inlet-2 & $\lambda_{T}$ & 0.188 & 0.127 & 0.131 \\
\hline Rear Cavity & Rinlet-1 & $\lambda_{T}$ & 0.186 & 0.136 & 0.201 \\
\hline Drive Cone Cavity & Dinlet-1 & $\lambda_{T}$ & 0.143 & 0.121 & 0.104 \\
\hline \multirow{6}{*}{ Bore Cavity } & Binlet-1 & $\lambda_{T}$ & 0.245 & 0.407 & 0.353 \\
\hline & Binlet-2 & $\lambda_{T}$ & 0.341 & 0.662 & 0.600 \\
\hline & Bseal-inlet-1 & $\lambda_{T}$ & 0.012 & 0.010 & 0.020 \\
\hline & Bseal-inlet-2 & $\lambda_{T}$ & 0.017 & 0.023 & 0.021 \\
\hline & Bseal-inlet-3 & $\lambda_{T}$ & 0.016 & 0.022 & 0.021 \\
\hline & Bleak-inlet & $\lambda_{T}$ & 0.006 & 0.010 & 0.012 \\
\hline
\end{tabular}

Table 1 Boundary conditions for the CFD models 
Title: Coupled FE-CFD Thermal Analysis for a Cooled Turbine Disk Journal of Mechanical Engineering Science (Part C, Proc. IMechE)

\section{Figures.}

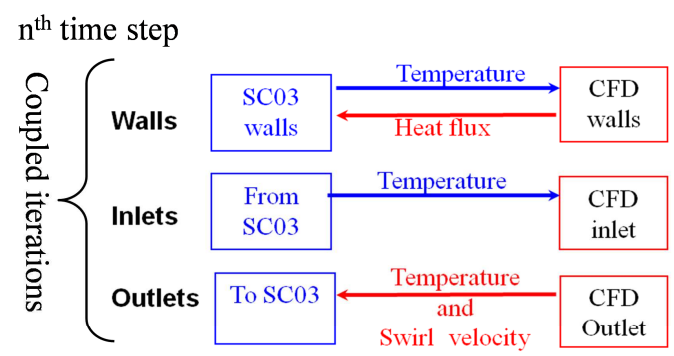

Following convergence progress to $\mathrm{n}+1^{\text {th }}$ time step

Figure 1 Coupling method

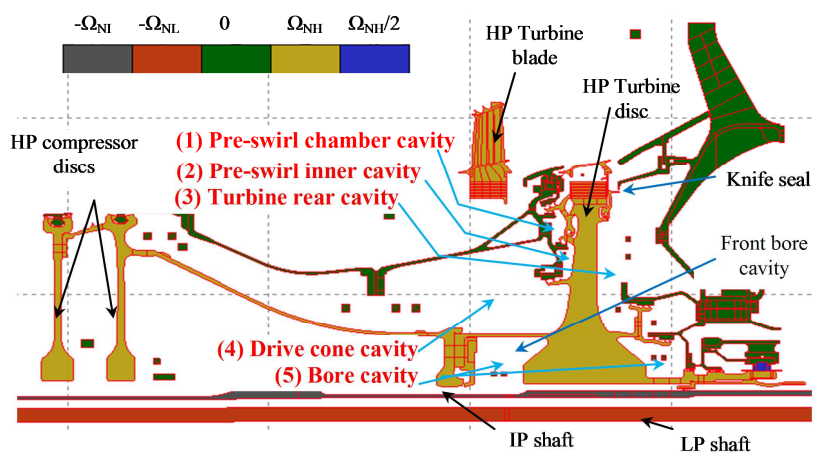

Figure 2 Axisymmetric HPT FE model

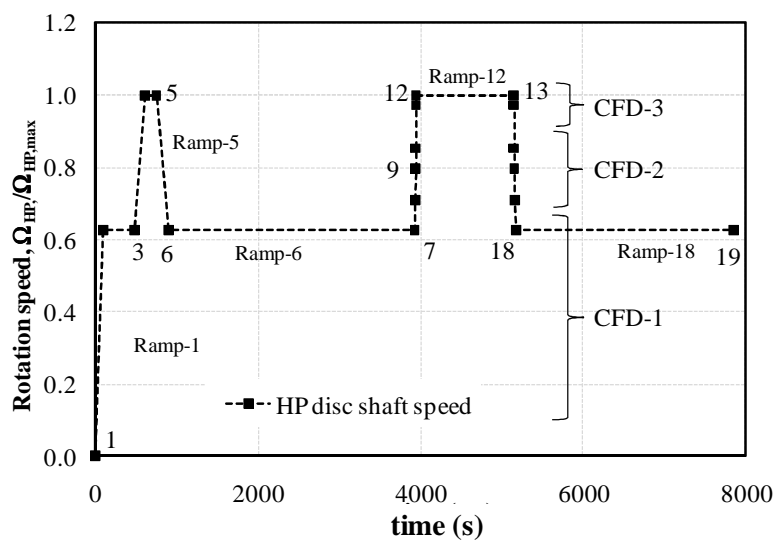

Figure 3 Transient cycle for HP turbine shaft 
Title: Coupled FE-CFD Thermal Analysis for a Cooled Turbine Disk Journal of Mechanical Engineering Science (Part C, Proc. IMechE)

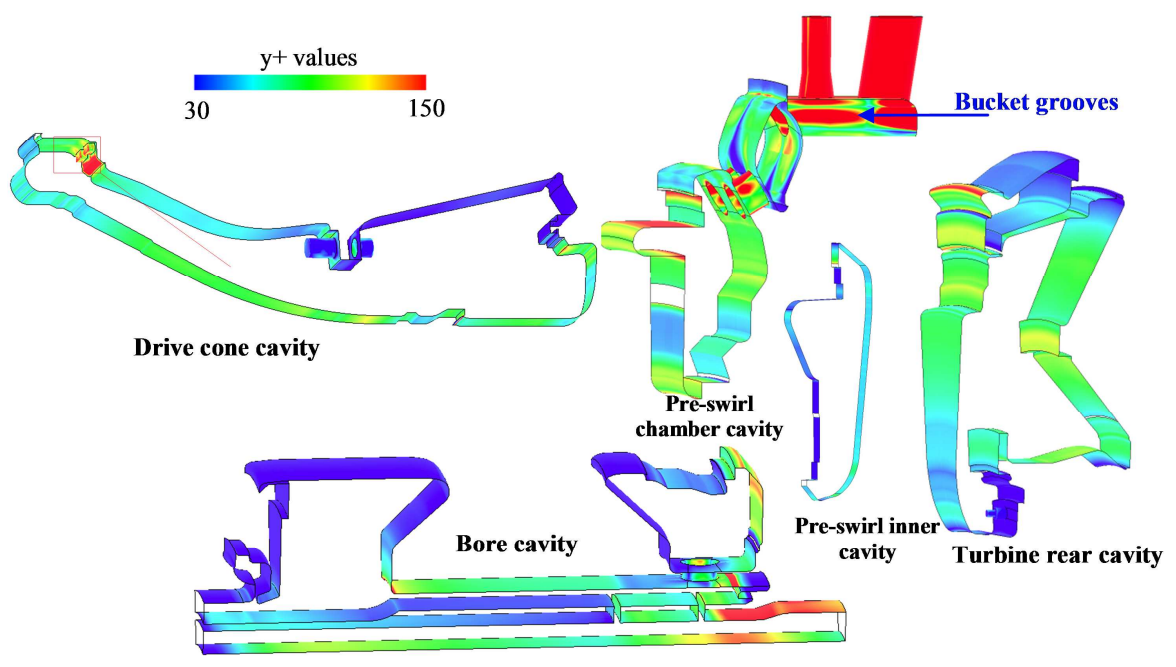

Figure 4 y+ values for 3D models for maximum power condition from Hydra

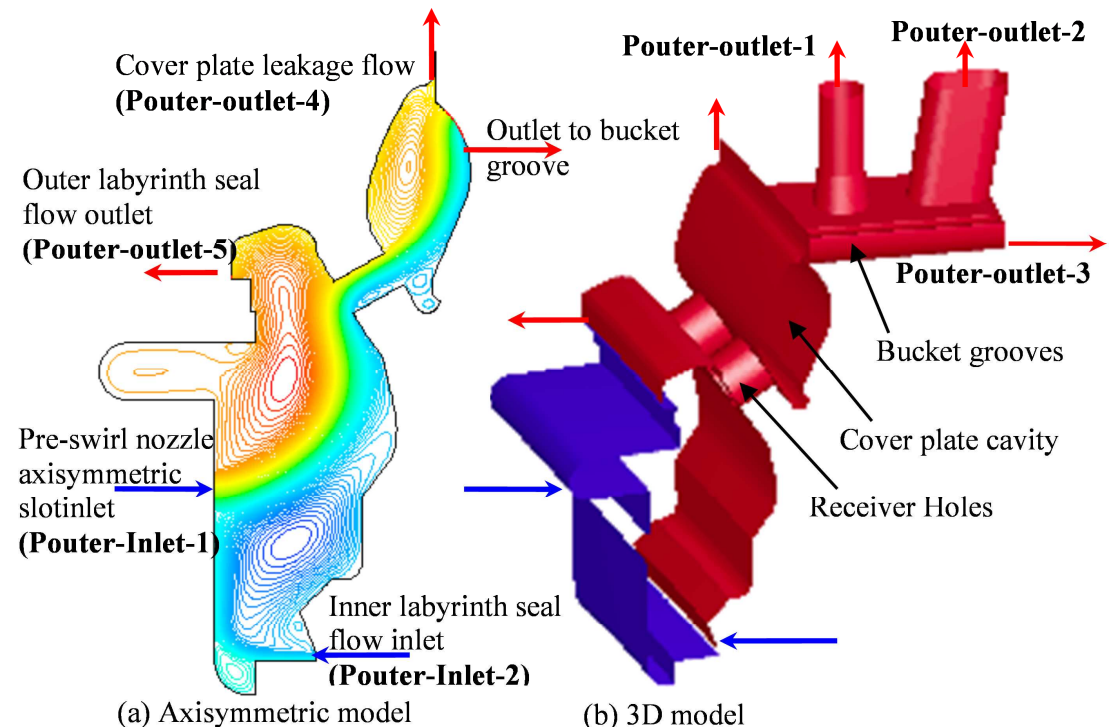

(a) Axisymmetric model

(b) $3 \mathrm{D}$ model

Figure 5 Pre-swirl outer cavity CFD models 
Title: Coupled FE-CFD Thermal Analysis for a Cooled Turbine Disk Journal of Mechanical Engineering Science (Part C, Proc. IMechE)
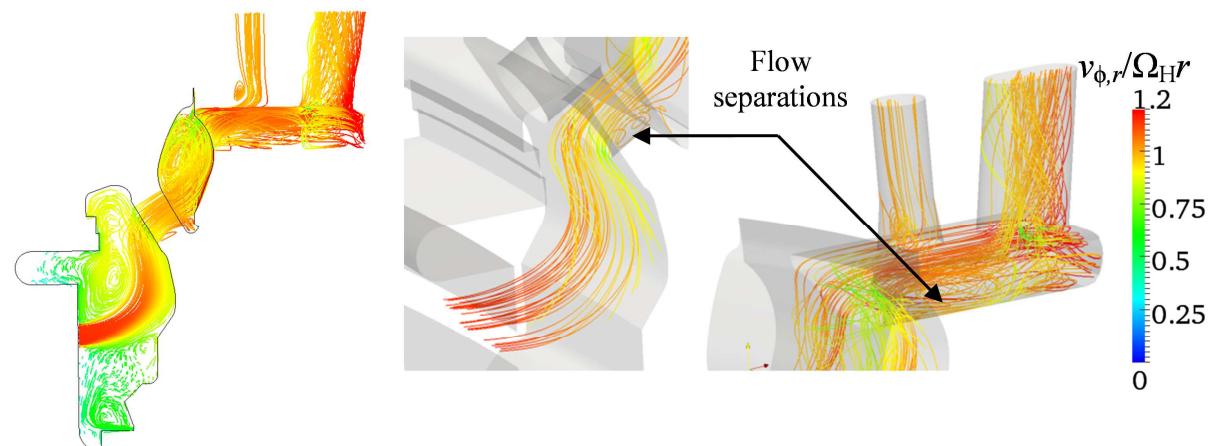

Figure 6 Path lines inside the 3D pre-swirl cavity

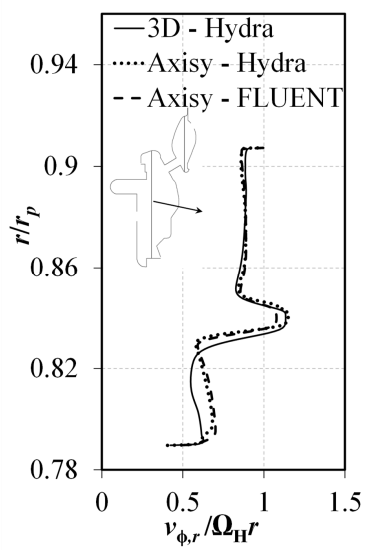

(a)

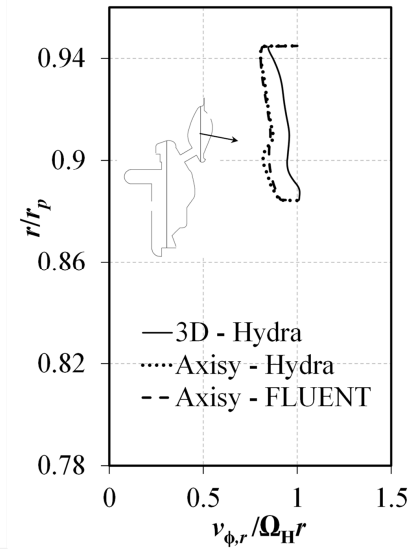

(b)

Figure 7 Swirl velocity in pre-swirl and cover plate cavities

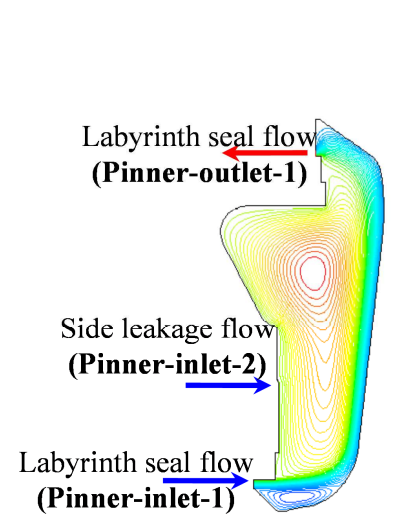

(a) Axisymmetric model

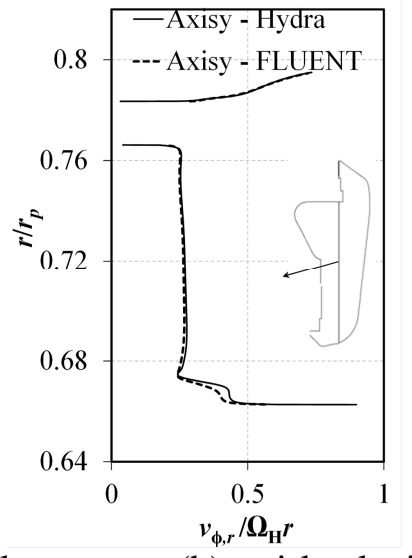

(b) swirl velocity

Figure 8 Pre-swirl inner cavity CFD models and swirl velocity for maximum power condition 
Title: Coupled FE-CFD Thermal Analysis for a Cooled Turbine Disk Journal of Mechanical Engineering Science (Part C, Proc. IMechE)

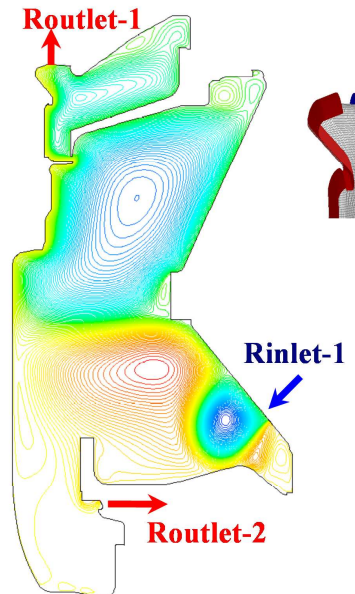

(a) Axisymmetric model

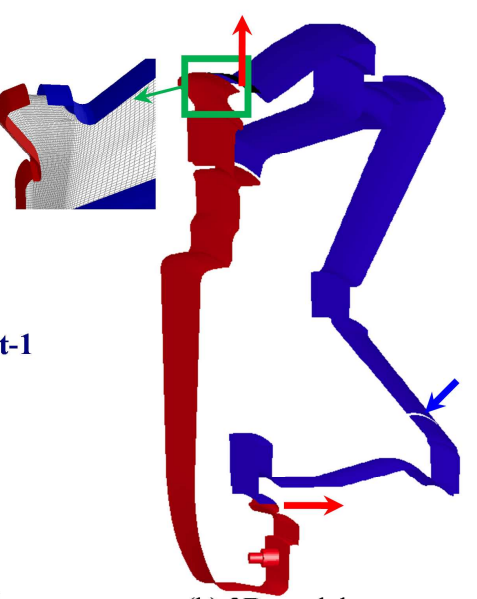

(b) 3D model

Figure 9 Turbine rear cavity CFD models

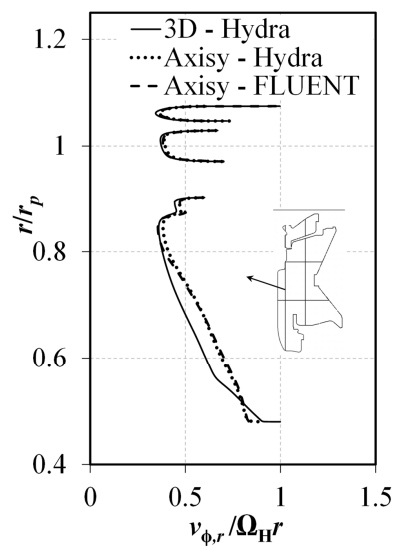

(a) Radial line-1

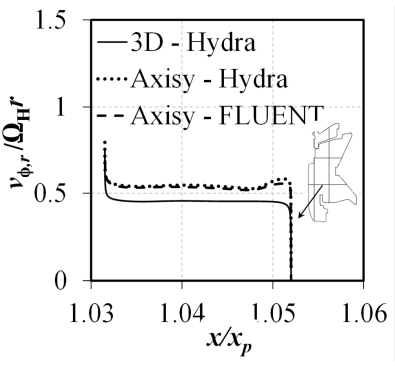

(b) Axial line-2

Figure 10 Swirl velocity in the turbine rear cavity for maximum power condition 
Title: Coupled FE-CFD Thermal Analysis for a Cooled Turbine Disk Journal of Mechanical Engineering Science (Part C, Proc. IMechE)

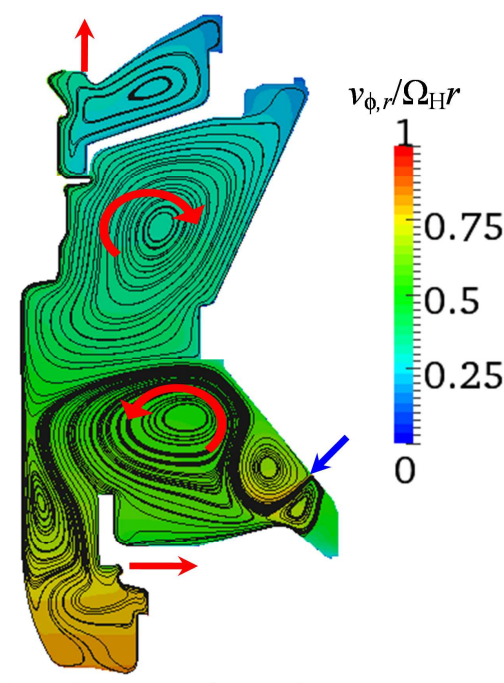

(a) Axisymmetric model

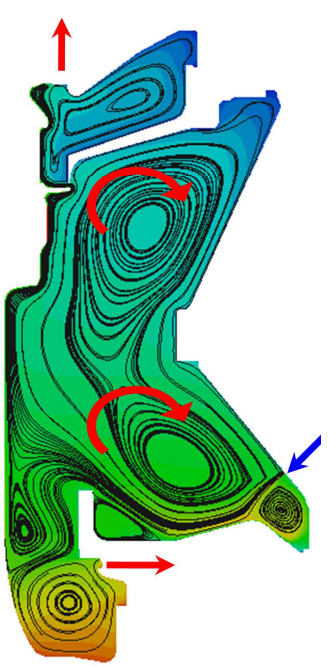

(b) 3D model

Figure 11 Flow structure (path lines) in the turbine rear cavity at maximum power condition from Hydra

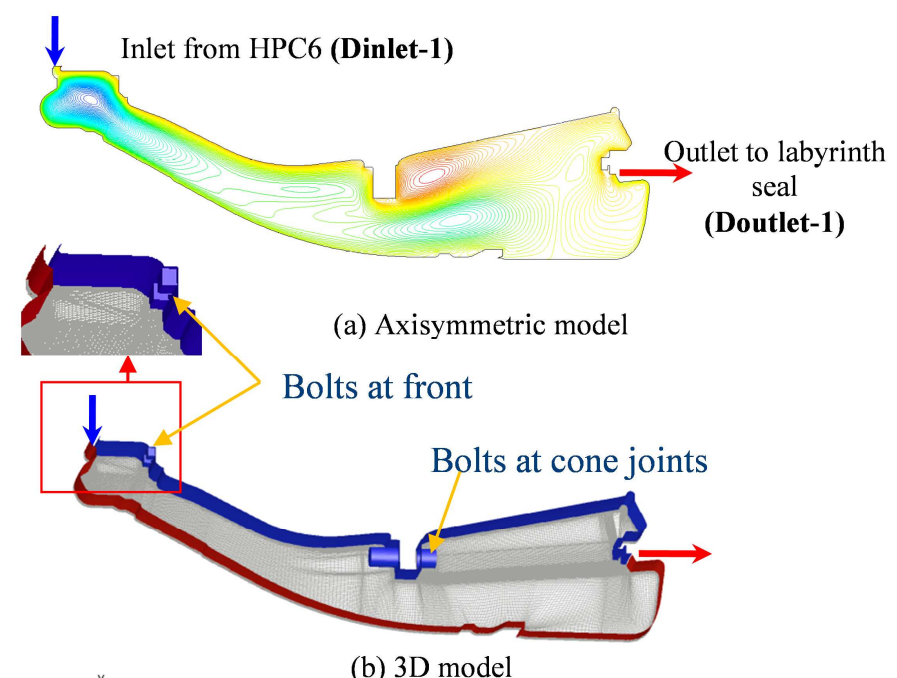

Figure 12 Drive cone cavity CFD models 
Title: Coupled FE-CFD Thermal Analysis for a Cooled Turbine Disk Journal of Mechanical Engineering Science (Part C, Proc. IMechE)

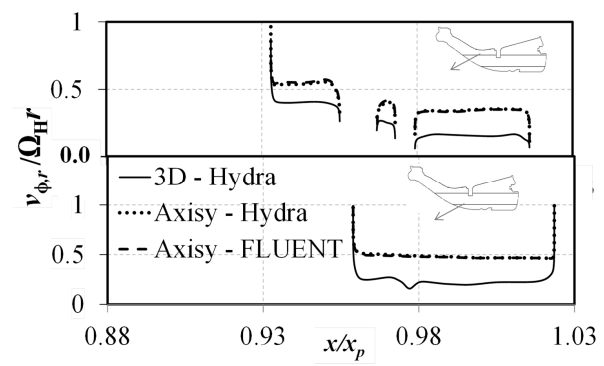

Figure 13 Swirl velocity in the drive cone cavity for maximum power

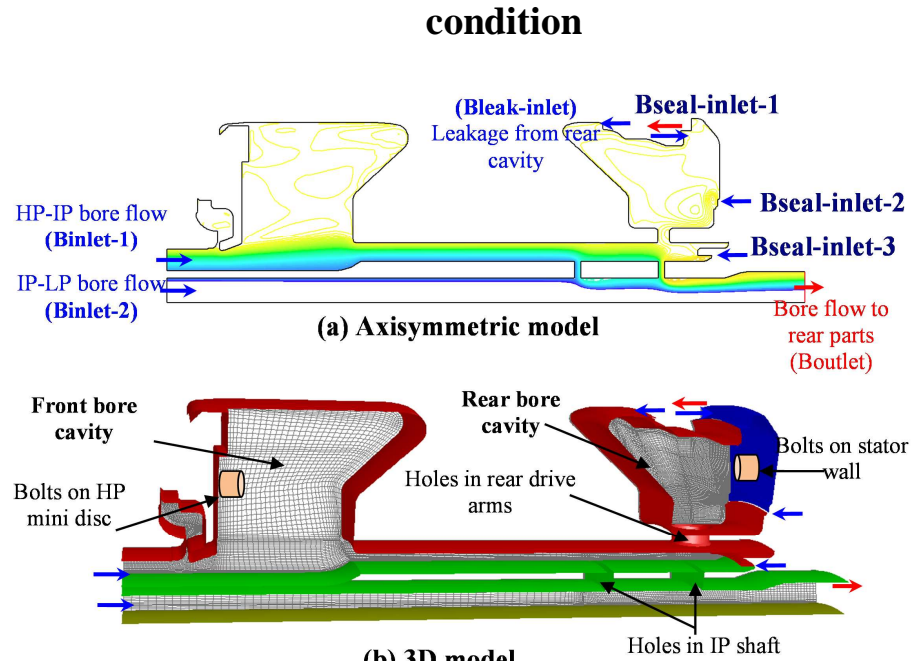

(b) 3D model

Holes in IP shaft

Figure 14 Bore cavity CFD models

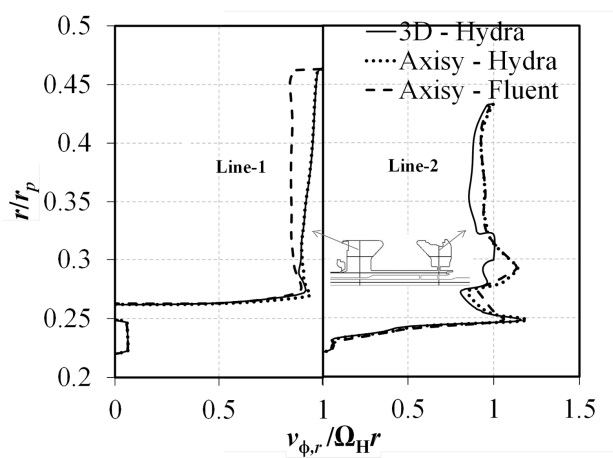

(a) Radial lines

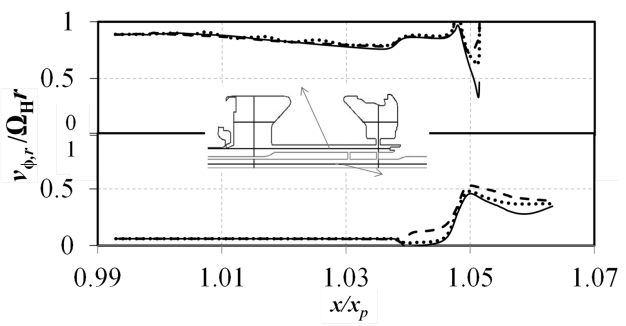

(b) Axial lines

Figure 15 Swirl velocity inside the bore cavity at maximum power condition 
Title: Coupled FE-CFD Thermal Analysis for a Cooled Turbine Disk Journal of Mechanical Engineering Science (Part C, Proc. IMechE)

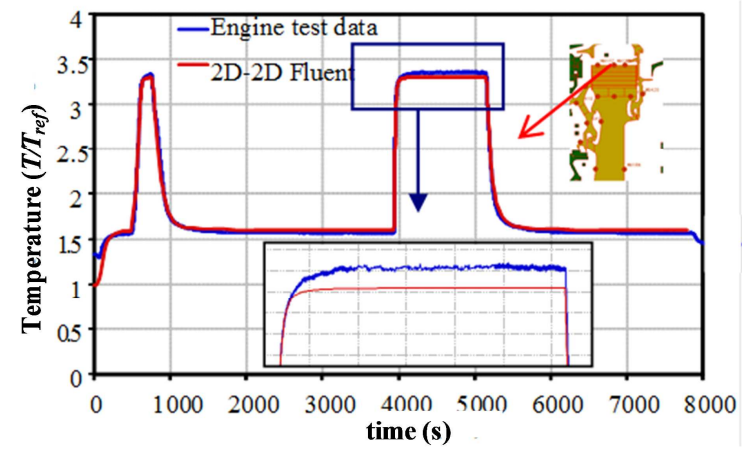

Figure 16 Calculated temperature and engine test data at reference point TCFO
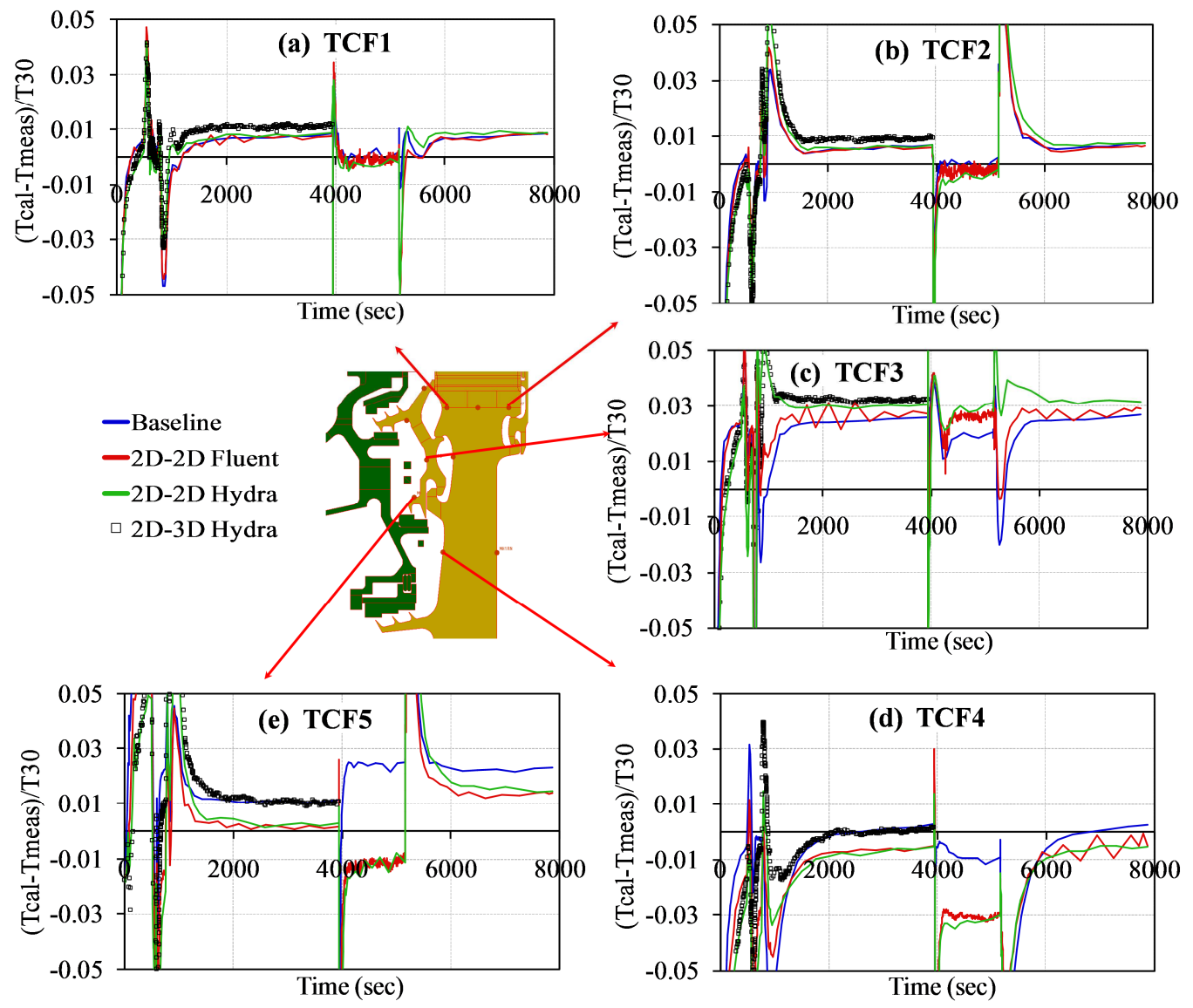

Figure 17 Reference point temperatures compared to engine test data in pre-swirl cavities and bucket groove 
Title: Coupled FE-CFD Thermal Analysis for a Cooled Turbine Disk Journal of Mechanical Engineering Science (Part C, Proc. IMechE)
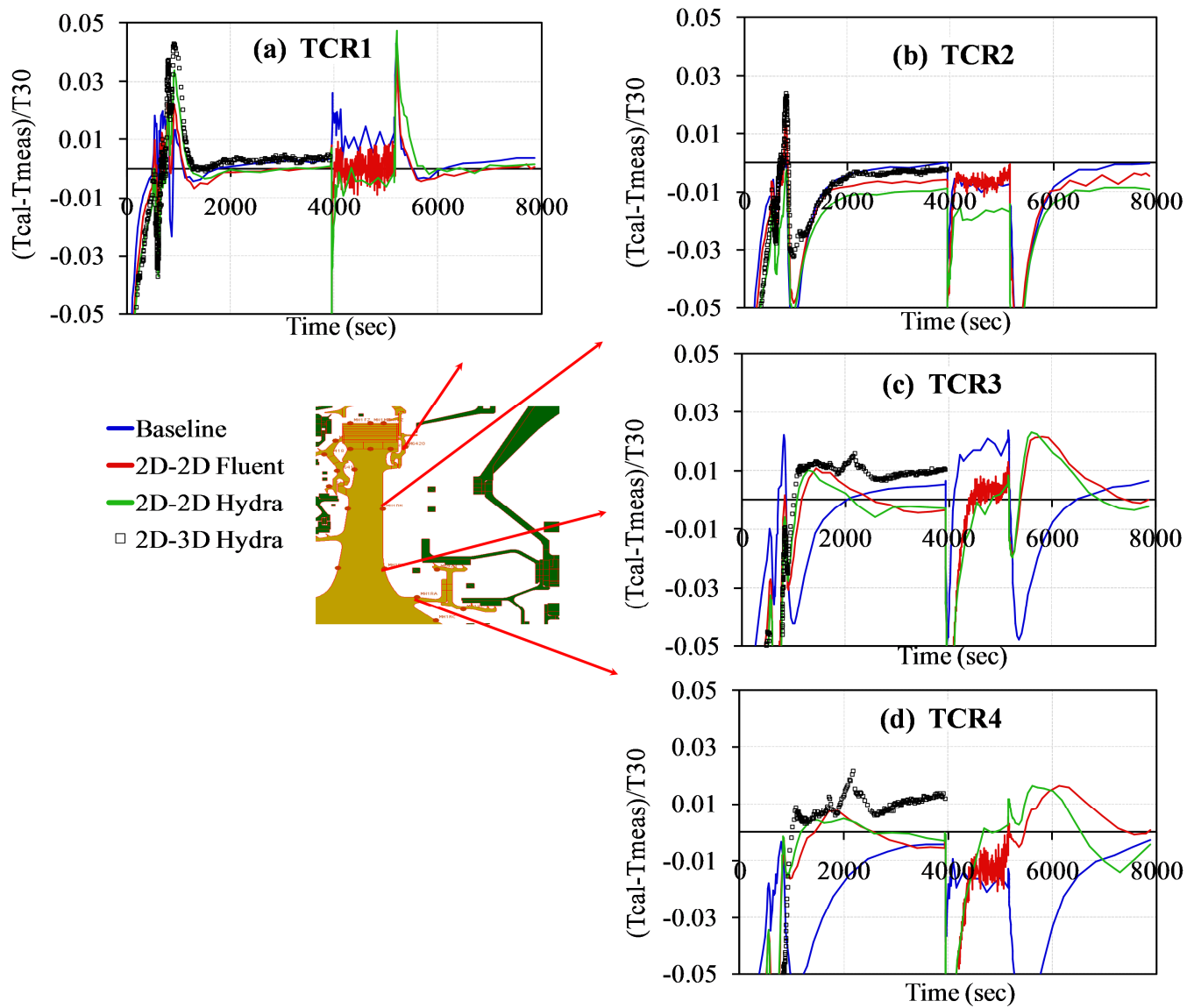

Figure 18 Reference point temperatures compared to Engine test data in rear cavity 
Title: Coupled FE-CFD Thermal Analysis for a Cooled Turbine Disk Journal of Mechanical Engineering Science (Part C, Proc. IMechE)
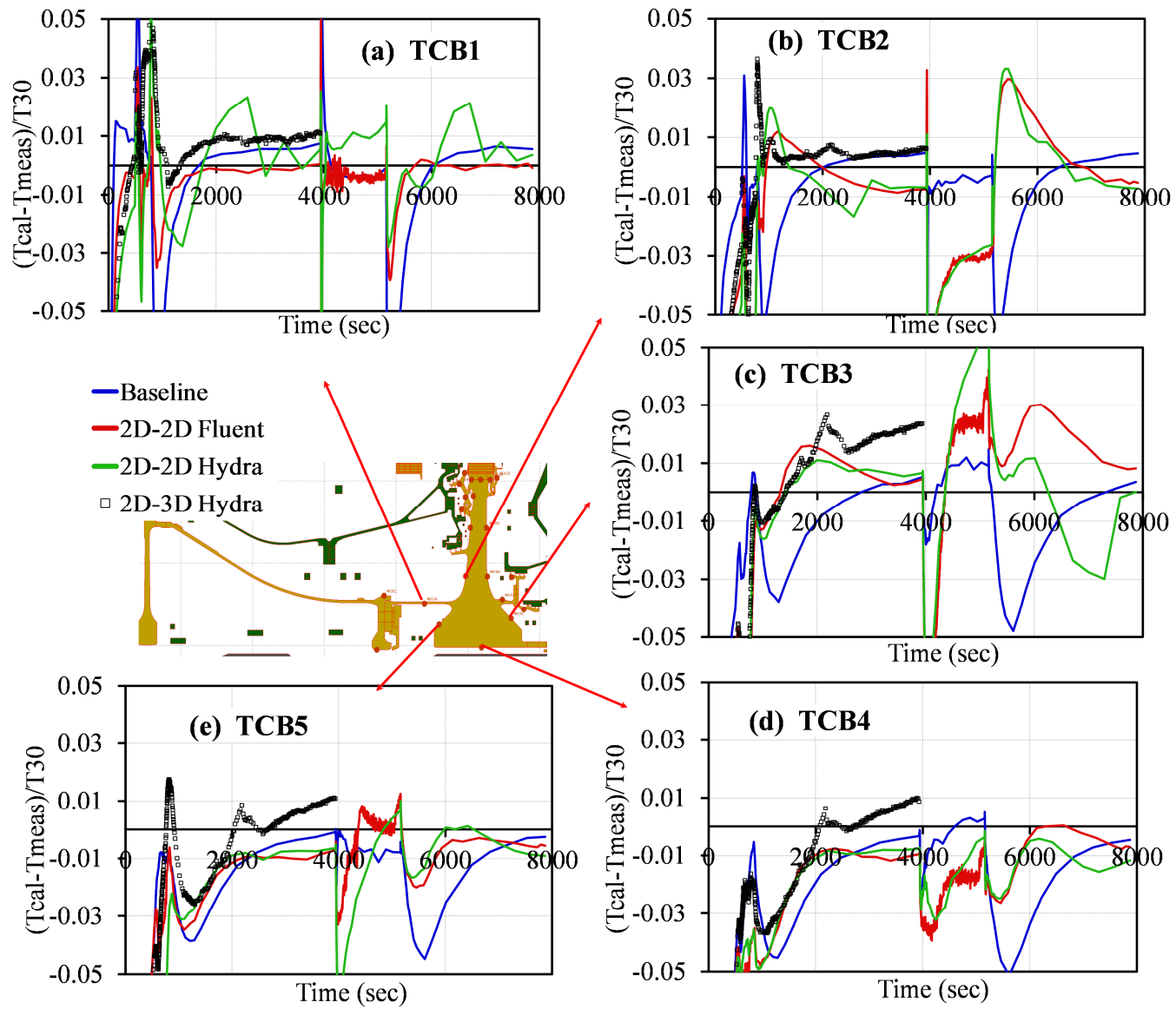

Figure 19 Reference point temperatures compared to engine test data in drive cone cavity and bore cone cavities 
Title: Coupled FE-CFD Thermal Analysis for a Cooled Turbine Disk Journal of Mechanical Engineering Science (Part C, Proc. IMechE)

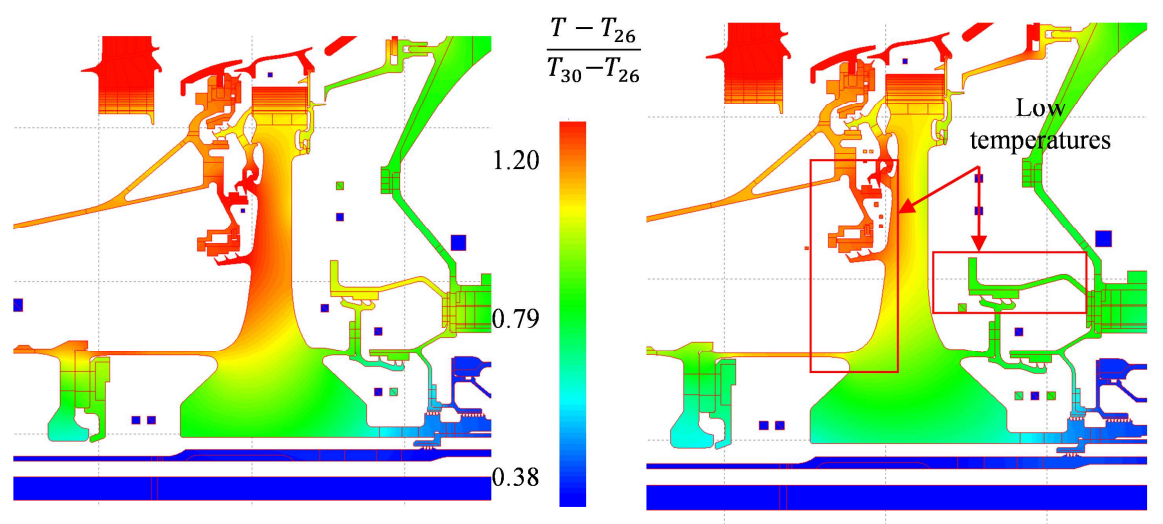

(a) Baseline (b) 2D-2D Hydra

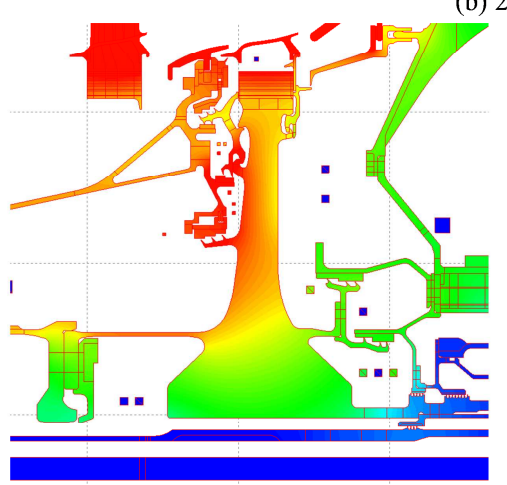

(c) 2D-3D Hydra

Figure 20: Temperature contour plots at low power, time $=2600 s \& \operatorname{Re}_{\phi}=1.44 \times 10^{6}$

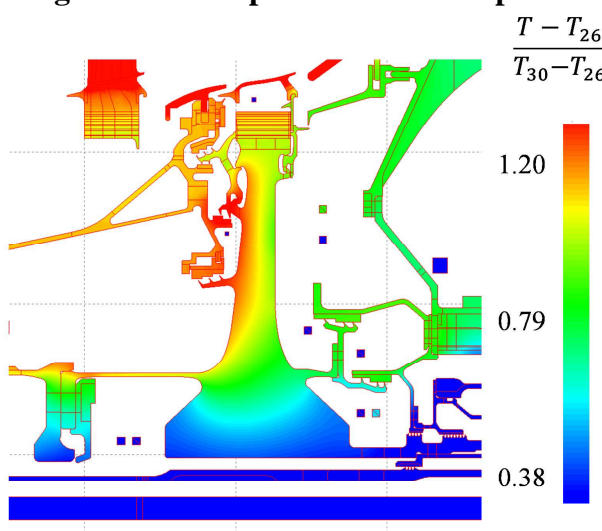

(a) Baseline

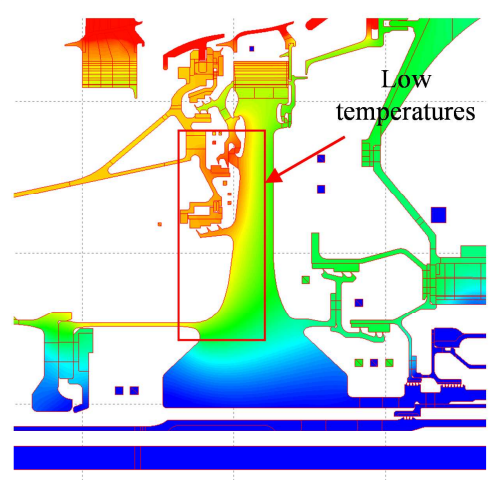

(b) 2D-2D Hydra

Figure 21: Temperature contour plots at maximum power, time $=4600 s \& \mathrm{Re}_{\phi}=4.03 \times 10^{7}$ 